\title{
Distortions in Power Spectra
}

\author{
Ilona Merke and Helmut Dreizler
}

Abteilung Chemische Physik im Institut für Physikalische Chemie der Universität Kiel

Z. Naturforsch. 43a, 196-202 (1988); received December 11, 1987

Power spectra are commonly used in microwave Fourier transform spectroscopy. We point out distortions, which may lead to erroneous frequency measurements.

\section{Introduction}

The spectra of microwave Fourier transform (MWFT) spectroscopy [1-3] are usually calculated in the form of power spectra [4]. For the line frequencies the peak values were taken. It was noticed that the maxima $\bar{v}_{i}$ of a multiplet pattern do not agree with the true frequencies $v_{i}$, of the multiplet components $i$, as there is a complicated distortion of the shape of the multiplet. It became evident by comparison with theory [5] and comparison with other spectroscopic techniques [6] that this procedure should be changed.

Therefore we consider the influence of the amplitude $A^{(i)}$, the phase $\varphi^{(i)}$ and the relaxation time $T_{2}^{(i)}$ of the component $i$ on its displayed frequency $\bar{v}_{i}$.

\section{Mathematical Background}

For the mathematical formulation we used the discrete Fourier transform formalism [7,8] as this is best adapted to the digital measuring technique.

According to theoretical considerations [9] and experimental observations the signal of a single line or component of a multiplet in the time domain is a damped sine function:

$$
X^{(i)}(t)=A^{(i)}\left[\sin \left(2 \pi m^{(i)} v_{0} t+\varphi^{(i)}\right)\right] \exp \left(-t / T_{2}^{(i)}\right) .
$$

We omit Doppler broadening in these considerations. For simplicity we have assumed in (1), that the frequency is exactly

$$
v^{(i)}=m^{(i)} v_{0}=m^{(i)} \cdot 1 / T
$$

Reprint requests to Prof. Dr. H. Dreizler, Abteilung Chemische Physik im Institut für Physikalische Chemie, ChristianAlbrechts-Universität, Olshausenstr. 40, D-2300 Kiel, FRG. with $T$ the total measuring time of the time domain signal. Thereby the leakage effect [10] is eliminated without losing generality.

As the signal is measured by a digital technique, the time $t$ is restricted to the values $n T / 2 N$ at $2 N$ measuring or sampling points with $0 \leq n \leq 2 N-1$.

The discrete Fourier transformation of a single component $i$ is

$$
\begin{aligned}
A^{(i)}(k)= & (1 / 2 N) \sum_{n=0}^{2 N-1} X^{(i)}(n T / 2 N) \\
& \cdot \exp (-i 2 \pi n k / 2 N), 0 \leq k \leq 2 N-1 .
\end{aligned}
$$

A discrete Fourier series synthesizes the time domain signal at $2 \mathrm{~N}$ measuring points

$$
\begin{aligned}
& \mathrm{X}^{(i)}(n T / 2 N)=\sum_{k=0}^{2 N-1} A^{(i)}(k) \exp (i 2 \pi n k / 2 N), \\
& 0 \leq n \leq 2 N-1 .
\end{aligned}
$$

The complex Fourier coefficients $A^{(i)}(k)$ and the time domain signal $X^{(i)}(n T / 2 N)$ are functions with the pe$\operatorname{riod} 2 N$. The $A^{(i)}(k)$ may be split into coefficients $\mathrm{a}_{k}^{(i)}$ and $b_{k}^{(i)}$ of the real Fourier transformation:

$$
\begin{aligned}
& A^{(i)}(k)=(1 / 2)\left(a_{k}^{(i)}-i b_{k}^{(i)}\right), \\
& 0 \leq k \leq 2 N-1,
\end{aligned}
$$

with

$$
\begin{aligned}
& a_{k}^{(i)}=(1 / N) \sum_{n=0}^{2 N-1} X^{(i)}(n T / 2 N) \cos (2 \pi n k / 2 N), \\
& 0 \leq k \leq N, \\
& b_{k}^{(i)}=(1 / N) \sum_{n=0}^{2 N-1} X^{(i)}(n T / 2 N) \sin (2 \pi n k / 2 N), \\
& 1 \leq k \leq N-1, \quad b_{0}^{(i)}=b_{N}^{(i)}=0 .
\end{aligned}
$$

Observe that (6) can be obtained directly by a real discrete Fourier transformation. Further it can be

0932-0784/88/0300-0196 $\$ 01.30 / 0$. Please order a reprint rather than making your own copy. 
seen, that the following relations are valid:

$$
\begin{aligned}
& a_{k}^{(i)}=a_{-k}^{(i)}=a_{k+j 2 N}^{(i)}=a_{-k+j 2 N}^{(i)}, \\
& b_{k}^{(i)}=-b_{-k}^{(i)}=b_{k+j 2 N}^{(i)}=-b_{-k+j 2 N}^{(i)},
\end{aligned}
$$

with $j$ a positive or negative integer. The $k$ range is extended for these relations. With (7) it is obvious, that there is a redundancy in the $A^{(i)}(k)$ :

$$
A^{(i)}(k)=A^{(i)}(2 N-k)^{*}
$$

Further, from (7) and ( $8 \mathrm{a})$ :

$$
\begin{aligned}
A^{(i)}(k) & =A^{(i)}(k+j 2 N)=\left(A^{(i)}(-k)\right)^{*} \\
& =\left(A^{(i)}(-k+j 2 N)\right)^{*},
\end{aligned}
$$

A part of the relations (8) expresses the periodicity of (3). By (8) $N+1$ values of the $A^{(i)}(k)$ remain, which contain $2 N$ pieces of information, as $A^{(i)}(0)$ $=A^{(i)}(2 N)$ and $A^{(i)}(N)$ are real. This confirms the fact that from $2 N$ measurements $2 N$ pieces of information can be obtained and displayed in the form of a power spectrum [4],

$$
P_{k}=\left(a_{k}^{(i)}\right)^{2}+\left(b_{k}^{(i)}\right)^{2} .
$$

Because of the redundancy in most calculations only the spectral points with $0 \leq k \leq N$ are displayed.

For (1) the discrete Fourier transformation may be calculated by a discrete convolution of the Fourier transform of the sine- and exponential function of (1) [11].

The result is

$$
\begin{aligned}
& a_{k}^{(i)}=\left(A^{(i)} / 2\right)\left\{\left[a_{e}^{(i)}\left(k-m^{(i)}\right)+a_{e}^{(i)}\left(k-2 N+m^{(i)}\right)\right]\right. \\
& \cdot \sin \varphi^{(i)}-\left[b_{e}^{(i)}\left(k-m^{(i)}\right)\right. \\
&-\left.\left.b_{e}^{(i)}\left(k-2 N+m^{(i)}\right)\right] \cos \varphi^{(i)}\right\} \\
& b_{k}^{(i)}=\left(A^{(i)} / 2\right)\left\{\left[b_{e}^{(i)}\left(k-m^{(i)}\right)+b_{e}^{(i)}\left(k-2 N+m^{(i)}\right)\right]\right. \\
& \cdot \sin \varphi^{(i)}+\left[a_{e}^{(i)}\left(k-m^{(i)}\right)\right. \\
&\left.\left.-a_{e}^{(i)}\left(k-2 N+m^{(i)}\right)\right] \cos \varphi^{(i)}\right\}
\end{aligned}
$$

with

$$
\begin{aligned}
a_{e}^{(i)}(l)= & (1 / D) \cdot\left[1-\exp \left(-T / T_{2}^{(i)}\right)\right] \\
& \cdot\left[1-\exp \left(-T / 2 N T_{2}\right) \cos (2 \pi l / 2 N)\right], \\
b_{e}^{(i)}(l)= & (1 / D) \cdot\left[1-\exp \left(-T / T_{2}^{(i)}\right)\right] \quad(11 \mathrm{~b}) \\
& \cdot\left[\exp \left(-T / 2 N T_{2}\right) \sin (2 \pi l / 2 N)\right], \\
D=N & {\left[1-2 \exp \left(-T / 2 N T_{2}^{(i)}\right) \cos (2 \pi l / 2 N)\right.} \\
+ & \left.\exp \left(-T / N T_{2}^{(i)}\right)\right],
\end{aligned}
$$

if the sine function has the frequency $m^{(i)} v_{0}$. The coefficients (10) with (11) fulfil the conditions (7) and (8).

\section{Distortion of a Single Line}

In Fig. 1 is illustrated, that a line with a frequency $m^{(i)} v_{0}$ near the edge $k=0$ or $k=N$ of the spectral
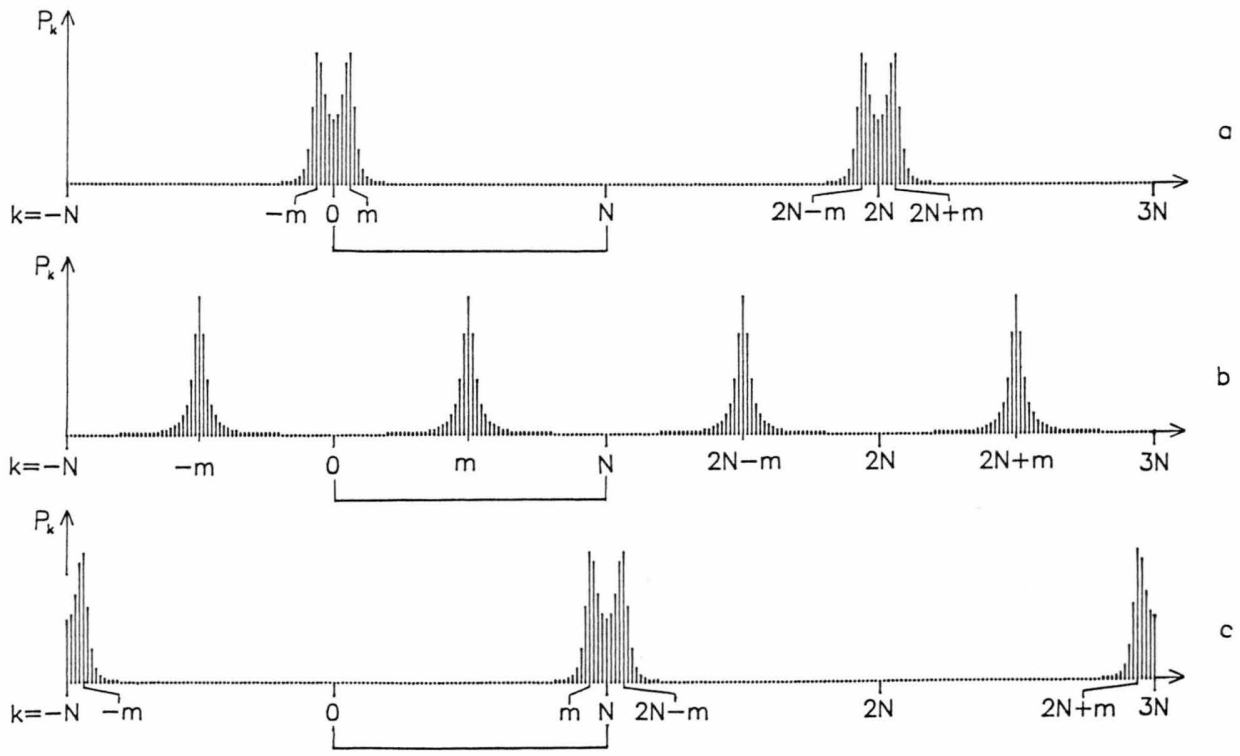

Fig. 1. Distortion of the lineshape of the power spectrum of a line in three different positions in the spectral range. The displayed window is indicated. The number of datapoints was reduced for reasons of the graphic display. (64 data points $=10 \mathrm{MHz}$ ). $2 N=128, \varphi=0, \Delta v_{1 / 2}=250 \mathrm{kHz} \approx 2$ data points; a) $v=4 v_{0}$; b) $v=32 v_{0}$; c) $v=60 v_{0}$. 
range $0 \leq k \leq N$ is distorted. $N v_{0}$ is the Nyquist frequency.

We consider now in more detail by numerical calculations the influence of the frequency $v=m v_{0}$, the amplitude $\mathrm{A}$, the phase $\varphi$ and the linewidth $\Delta v_{1 / 2}=1 / 2 \pi T_{2}(\mathrm{HWHH})$ on the value of the peak frequency $\bar{v}$ of a single line determined from the power spectrum by a fit of the three highest data points of the line contour to a Lorentzian line shape. The superscript $i$ has been omitted. Therefore we consider the spectrum resulting from (1). We take $2 N=1024$ recorded data points when we calculate the discrete Fourier transform by the same program as used in our spectrometers [13]. Zero filling [14] to 4096 data points is not included.

\section{Influence of the Line Frequency}

When the line frequency $m v_{0}$ is moved towards zero or the Nyquist frequency $N v_{0}$ a rapid increase of the deviation $\delta \bar{v}=v-\bar{v}$ of the frequency $\bar{v}$ is observed. If $\bar{v}$ is taken as the line frequency a systematic error $\delta \bar{v}$ is present. This is easily understood from (8) and the fact, that the Fourier transform of (1) contains not only the frequency of the sine function but also components in its neighbourhood (Figure 1). The magnitude of these components depends on the width of the considered line. A line centered near the Nyquist frequency (or the zero frequency) has frequency components $(N+k) v_{0}$ which lie beyond the limits of the spectral range. They are folded back inside the spectral range and appear at the positions $(N-k) v_{0}$, which causes an increase of the magnitude of the component at this spectral point and produces a distortion of the lineshape. This results in a deviation $\delta \bar{v}=v-\bar{v}$ of the maximum $\bar{v}$ of the line with respect to $v$. Figure 2 gives examples for three different $\Delta v_{1 / 2}$ near the zero frequency.

\section{Influence of the Phase}

Figure 3 shows the influence of the phase $\varphi$ on the error of the frequency determination. We choose a fixed frequency of $99.656 \mathrm{kHz}$ ( 5 datapoints) below the Nyquist frequency $N v_{0}$ and a line width $\Delta v_{1 / 2}=$ $20 \mathrm{kHz}$. The deviation varies sinusoidally with the phase $\varphi$. It should be noted that under experimental conditions $\varphi$ varies for a line at $10 \mathrm{MHz}$, the Nyquist frequency for 50 ns sample intervall, from 0 to $2 \pi$ by a delay time from 0 to $100 \mathrm{~ns}$. A further calculation is

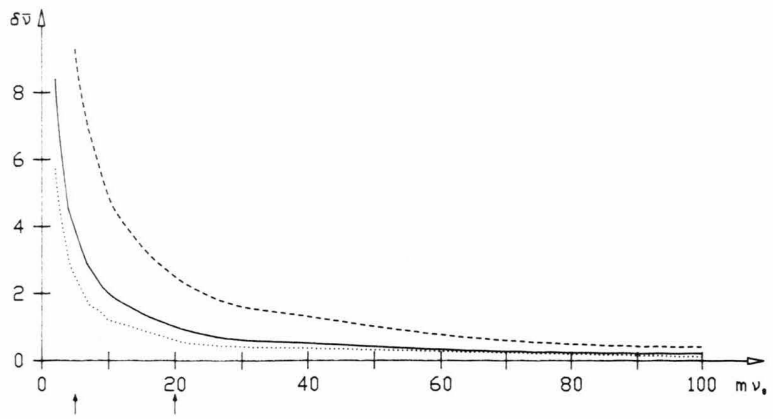

Fig. 2. Deviation $\delta \bar{v}[\mathrm{kHz}]$ of a line near zero frequency as a function of $v=m v_{0}$ expressed in multiples $m$ of $v_{0}=1 / \mathrm{T}$. $\varphi=0 ; \quad----: \Delta v_{1 / 2}=40 \mathrm{kHz} ; \quad-\Delta v_{1 / 2}=20 \mathrm{kHz}$ $\cdots \cdot \Delta v_{1 / 2}=10 \mathrm{kHz}$. An equal situation is near the Nyquist frequency, $\uparrow$ Line position for Figure 3 .

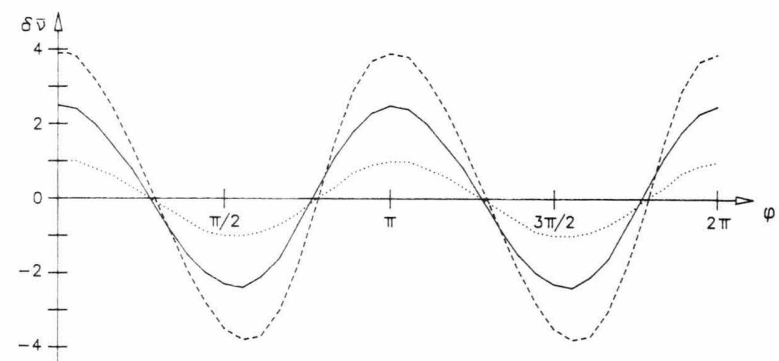

Fig. 3. Deviation $\delta \bar{v}[\mathrm{kHz}]$ of a line near the Nyquist frequency $N v_{0}$ as a function of the phase $\varphi .---: \Delta v_{1 / 2}=20 \mathrm{kHz}$; $\longrightarrow: \Delta v_{1 / 2}=10 \mathrm{kHz}, v=(N-5) v_{0} ; \cdots \cdot \Delta v_{1 / 2}=20 \mathrm{kHz}$, $v=(N-20) v_{0}$.

given for a line $398.624 \mathrm{kHz}$ (20 data points) below $N v_{0}$ in Figure 3.

It can be seen, that the deviation $\delta \bar{v}$ increases with the line width and by approaching $N v_{0}$. The same situation is near zero frequency.

\section{Influence of the Line Width}

The influence of the line width can be extracted from Fig. 2 and Fig. 3 as $\Delta v_{1 / 2}$ is parameter of the curves.

\section{Mathematical Background for Multiplets}

Next we consider multiplets with a special attention to the separation of its components, as this separation is often a valuable information. It is assumed that the components are sufficiently narrow and placed in the 
middle of the range $0 \leq k \leq N$, to minimize the edge distortions. Under these conditions we neglect terms with index $k-2 N+m^{(i)}$ of (10).

From the definition of the power spectrum (9) we obtain with (10) and (11)

$$
\begin{gathered}
P_{k}=(1 / 4)\left(\sum_{i=1}^{c} A^{(i)}\left[a_{e}^{(i)}\left(k-m^{(i)}\right) \sin \varphi^{(i)}\right)\right. \\
\left.\left.-b_{e}^{(i)}\left(k-m^{(i)}\right) \cos \varphi^{(i)}\right]\right)^{2} \\
+(1 / 4)\left(\sum _ { i = 1 } ^ { c } A ^ { ( i ) } \left[b_{e}^{(i)}\left(k-m^{(i)}\right) \sin \varphi^{(i)}\right.\right. \\
\left.\left.+a_{e}^{(i)}\left(k-m^{(i)}\right) \cos \varphi^{(i)}\right]\right)^{2}
\end{gathered}
$$

with $c$ the number of components. The summation of the $a_{k}^{(i)}$ and $b_{k}^{(i)}$ is valid, because the Fourier transformation is a linear operation. For a single line, $i=1$, (13) reduces to

$P_{k}=(1 / 4)\left(A^{(1)}\right)^{2}\left[\left(a_{e}^{(1)}\left(k-m^{(1)}\right)\right)^{2}+\left(b_{e}^{(1)}\left(k-m^{(1)}\right)\right)^{2}\right]$.

The important point is, that now the influence of the phase $\varphi^{(1)}$ vanishes in (14). This is the reason for the general use of the power spectra.

The phase $\varphi^{(i)}$ depends on the frequency of the component, the delay between the polarizing pulse and the starting point of the measurement and in a complicated way on the polarizing process by the strong MW-pulse.

For multiplets the influence of the phase $\varphi^{(i)}$ persists as may be seen more clearly by evaluating (13):

$$
\begin{aligned}
P_{k}= & (1 / 4) \sum_{i=1}^{c}\left(A^{(i)}\right)^{2}\left[\left(a_{e}^{(i)}\left(k-m^{(i)}\right)\right)^{2}+\left(b_{e}^{(i)}\left(k-m^{(i)}\right)\right)^{2}\right] \\
& +(1 / 2) \sum_{\substack{i=1 \\
i \neq j}}^{c} \sum_{i=1}^{c}\left\{A ^ { ( i ) } A ^ { ( j ) } \left[\left(a_{e}^{(i)}\left(k-m^{(i)}\right) a_{e}^{(j)}\left(k-m^{(j)}\right)\right.\right.\right. \\
& \cdot \cos \left(\varphi^{(i)}-\varphi^{(j)}\right) \\
& -a_{e}^{(i)}\left(k-m^{(i)}\right) b_{e}^{(j)}\left(k-m^{(j)}\right) \sin \left(\varphi^{(i)}-\varphi^{(j)}\right) \\
& +b_{e}^{(i)}\left(k-m^{(i)}\right) a_{e}^{(j)}\left(k-m^{(j)}\right) \sin \left(\varphi^{(i)}-\varphi^{(j)}\right) \\
& \left.\left.+b_{e}^{(i)}\left(k-m^{(i)}\right) b_{e}^{(j)}\left(k-m^{(j)}\right) \cos \left(\varphi^{(i)}-\varphi^{(j)}\right)\right]\right\} .
\end{aligned}
$$

From (15) it can be seen, that the absolute values of the phases loose their influence as only differences $\varphi^{(i)}-\varphi^{(j)}$ occur. The phase difference dependent terms become less important if $m^{(i)}$ and $m^{(j)}$ are sufficiently different from the spectral point of position $k$ referred to the line width of the components. Because the discussion of (15) in general terms is difficult we prefer to calculate examples with variation of amplitude $A^{(i)}$, frequency $m^{(i)} v_{0}$, phase $\varphi^{(i)}$ and relaxation time $T_{2}^{(i)}$ first for doublets to show the typical behaviour induced by the $i j$-terms in (15). The first line was chosen at the data point 256, the second above. All amplitudes are given as relative intensities $r=\left(A^{(i)} / A^{(j)}\right)^{2}$ in the power spectra.

\section{Influence of the Phase Difference}

The deviation $\delta(\Delta \bar{v})$ of the splitting $\Delta \bar{v}=\bar{v}_{1}-\bar{v}_{2}$ of the two components of a doublet from true values $v_{1}-v_{2}$ shows a strong dependence on the phase difference $\Delta \varphi=\varphi^{(i)}-\varphi^{(j)}$. In Fig. 4 we have chosen two lines separated by $78.125 \mathrm{kHz}$ (4 datapoints) of equal intensity $(r=1)$ and line width $\Delta v_{1 / 2}=20 \mathrm{kHz}$.

The largest deviation is approximately $16 \mathrm{kHz}$ or $21 \%$ of the splitting at $\Delta \varphi=0.31 \pi$ and $\Delta \varphi=\pi$. In MWFT spectroscopy a delay time of typical 0.3 to $3 \mu$ s between the end of the polarizing pulse and the beginning of the data recording is required for the attenuation of non-molecular perturbations. If the time domain signals originating from the two components are assumed to start with the same phase, the above delays produce a $\Delta \varphi$ from $0.05 \pi$ to $0.47 \pi$ for the example. Thus for realistic experimental conditions one works in the range of maximal deviations.

\section{Influence of the Intensity Ratio}

To study the influence of the intensity ratio $r$ on the splitting $\Delta \bar{v}=\bar{v}_{1}-\bar{v}_{2}$ we have chosen again a splitting $v_{1}-v_{2}=78.125 \mathrm{kHz}$. The phase difference was set $\Delta \varphi=0.31 \pi$ and the linewidth $\Delta v_{1 / 2}=20 \mathrm{kHz}$. The result is shown in Figure 5. In the range of $0.3 \leq r \leq 1$ the deviation $\delta(\Delta \bar{v})$ is nearly constant. Below $r=0.3$ it rises.

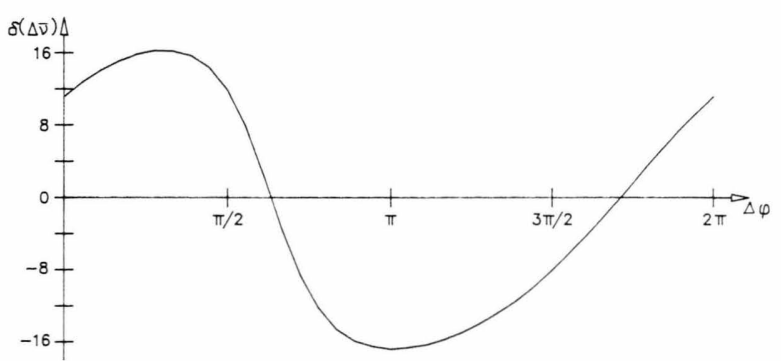

Fig. 4. Deviation $\delta(\Delta \bar{v})=\left(v_{1}-v_{2}\right)-\left(\bar{v}_{1}-\bar{v}_{2}\right)[\mathrm{kHz}]$ of the splitting $\bar{v}_{1}-\bar{v}_{2}$ of two components of a doublet from its true value $v_{1}-v_{2}$ as a function of $\Delta \varphi . v_{1}=256 v_{0}, v_{2}-260 v_{0}$, $\mathrm{A}^{(1)}=A^{(2)}, \Delta v_{1 / 2}=20 \mathrm{kHz}$. 


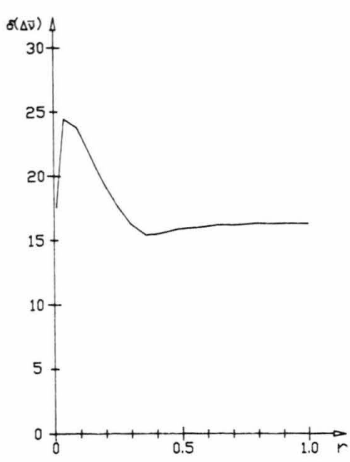

Fig. 5.

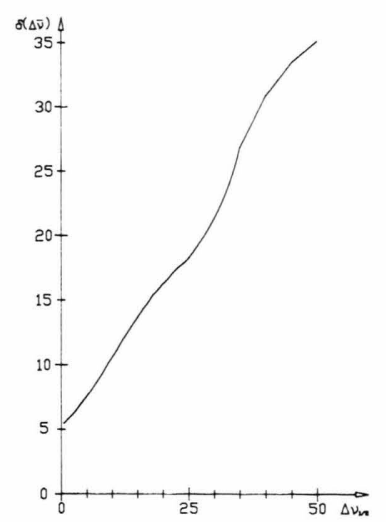

Fig. 6.
Fig. 5. Deviation $\delta(\Delta \bar{v})[\mathrm{kHz}]$ of the splitting $\bar{v}_{1}-\bar{v}_{2}$ of two components of a doublet from its true value $v_{1}-v_{2}$ as a function of $r=\left(A^{(1)} / A^{(2)}\right)^{2}, v_{1}=256 v_{0}, v_{2}=260 v_{0}$, $\Delta \varphi=0.31 \pi, \Delta v_{1 / 2}=20 \mathrm{kHz}$.

Fig. 6. Deviation $\delta(\Delta \bar{v})[\mathrm{kHz}]$ of the splitting $\bar{v}_{1}-\bar{v}_{2}$ of two components of a doublet from its true value $v_{1}-v_{2}$ as a function of $\Delta v_{1 / 2} ; v_{1}=256 v_{0}, v_{2}=260 v_{0}, \Delta \varphi=0.31 \pi$, $r=1$.
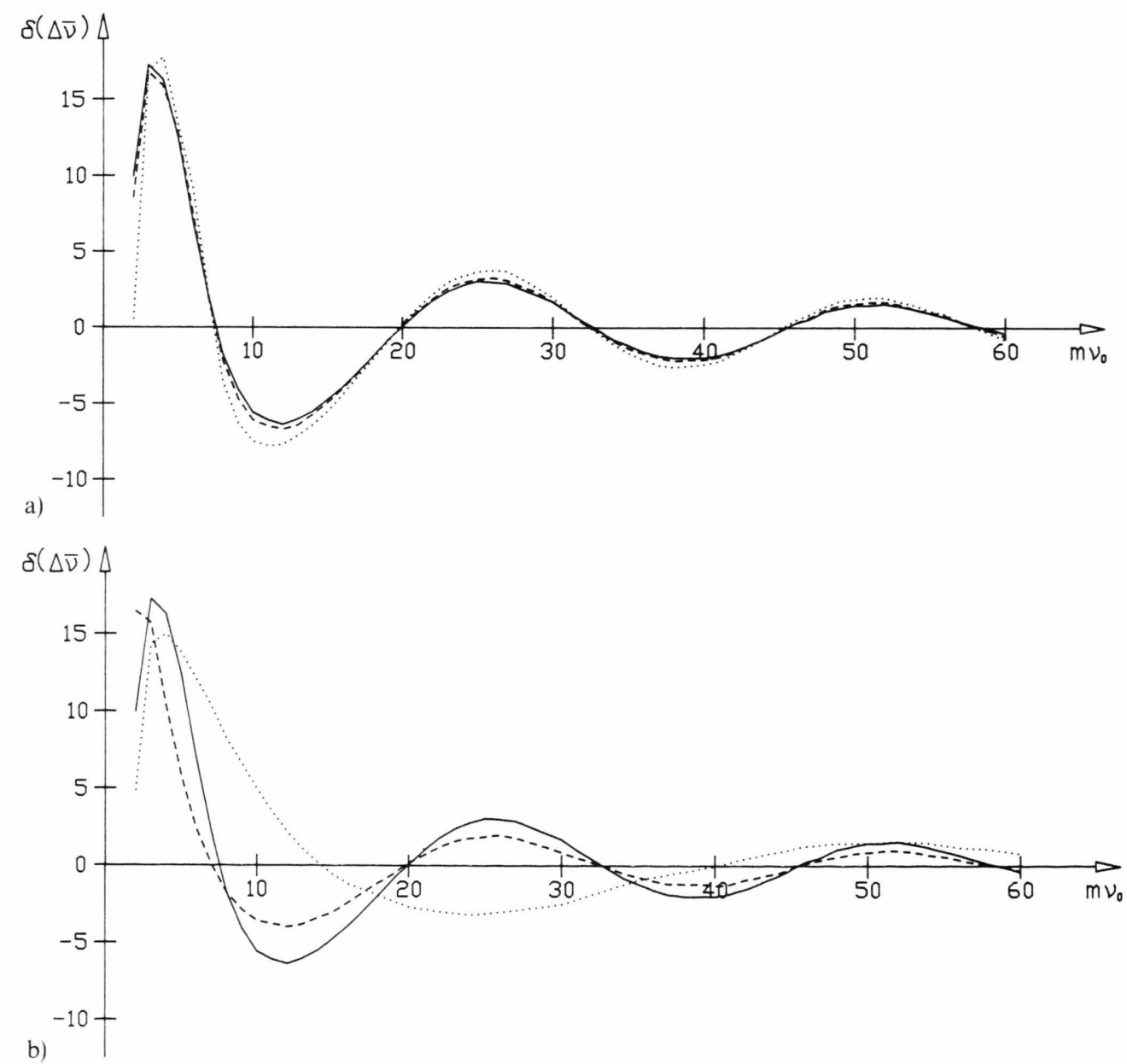

Fig. 7. Deviation $\delta(\Delta \bar{v})[\mathrm{kHz}]$ of the splitting $\Delta \bar{v}=\bar{v}_{1}-\bar{v}_{2}$ of a doublet as a function of the true splitting $v_{1}-v_{2}$. For fixed

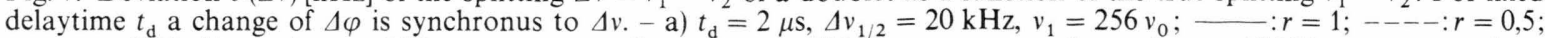
$\cdots r=0.25$. b) $r=1, v_{1}=256 v_{0} ;-: t_{\mathrm{d}}=2 \mu \mathrm{s}, \Delta v_{1 / 2}=20 \mathrm{kHz} ;---: t_{\mathrm{d}}=2 \mu \mathrm{s}, \Delta v_{1 / 2}=10 \mathrm{kHz} ; \cdots \cdot t_{\mathrm{d}}=1 \mu \mathrm{s}$, $\Delta v_{1 / 2}=20 \mathrm{kHz}$. 


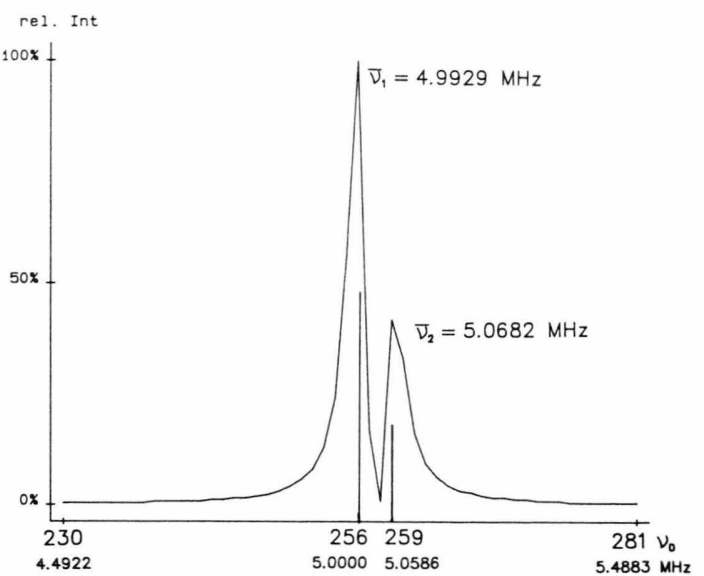

a)

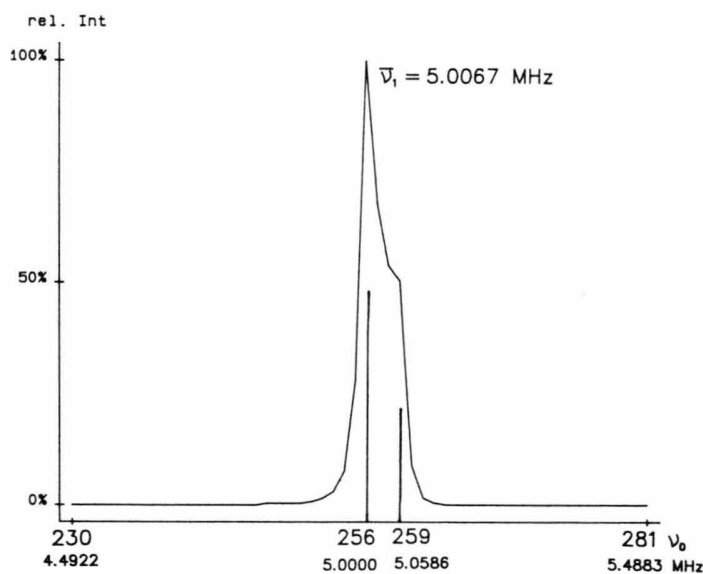

b)

Fig. 8. Powerspectrum of a doublet with $v_{1}=256 v_{0}$, $v_{2}=259 v_{0}, r=0.5, \Delta v_{1 / 2}=16 \mathrm{kHz}$. The frequencies $v_{i}$ and $\bar{v}_{\mathrm{i}}$ are given. The bar diagram was reduced by a factor of 0.5 . a) $\Delta \varphi=0.31 \pi$; b) $\Delta \varphi=\pi$.

\section{Influence of the Line Width}

Figure 6 demonstrates the dependence of the deviation $\delta(\Delta \bar{v})$ on the line width for a doublet separated $78.125 \mathrm{kHz}$ with $\Delta \varphi=0.31 \pi$ and $r=1$. It is plausible, that $\delta(\Delta \bar{v})$ increases with $\Delta v_{1 / 2}$ as the overlap of the components becomes more serious.

\section{Influence of the Splitting}

An increasing splitting reduces the overlap of the doublet components as does the narrowing of the line width. In Fig. 7 the splitting $\Delta v=v_{1}-v_{2}$ is varied

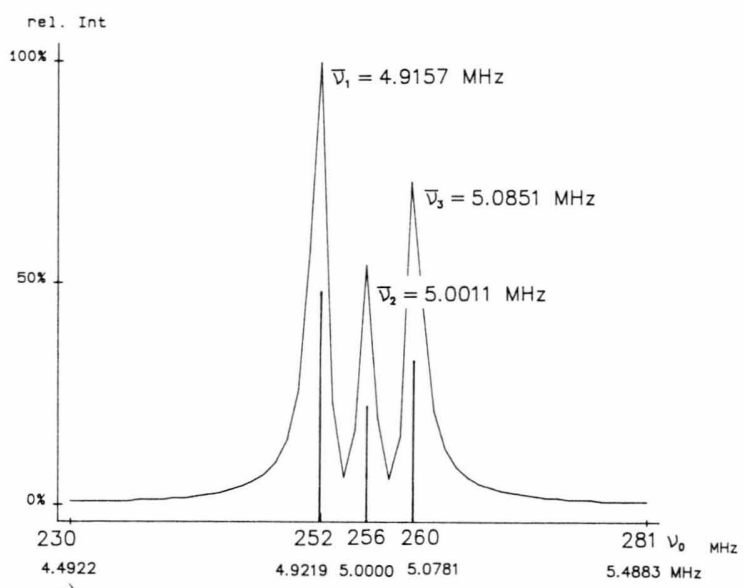

a)

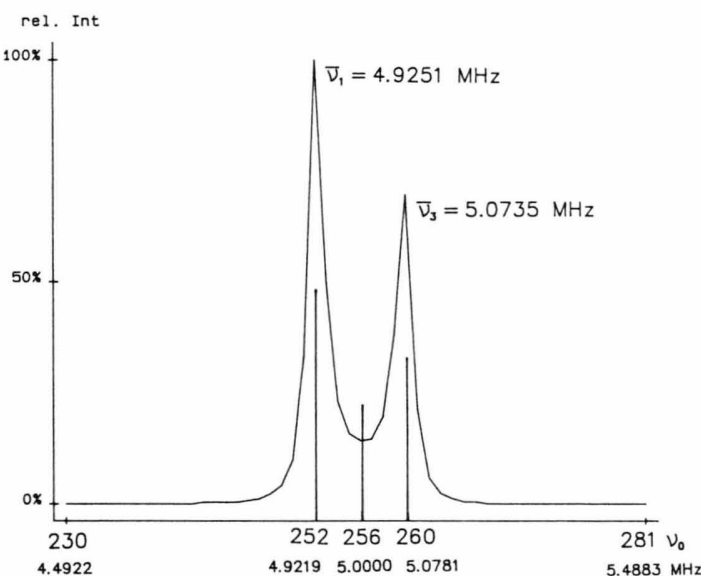

b)

Fig. 9. Powerspectrum of a triplett with $v_{1}=252 v_{0}$, $v_{2}=256 v_{0}, v_{3}=260 v_{0}, r_{12}=0.5, r_{13}=0.7, \Delta v_{1 / 2}=16 \mathrm{kHz}$. The frequencies $v_{i}$ and $\bar{v}_{i}$ are given. The bar diagram was reduced by a factor of 0.5 . a) $\Delta \varphi_{12}=\Delta \varphi_{23}=0$; b) $\Delta \varphi_{12}$ $=\Delta \varphi_{23}=0.61 \pi$

under constant $A^{(1)}=A^{(2)}$ and $\Delta v_{1 / 2}=20 \mathrm{kHz}$. As for a fixed delay time $t_{\mathrm{d}}$, which is chosen in a measurement as short as possible, $\Delta \varphi$ is related to $v_{1}-v_{2} . \Delta \varphi$ varies with

$$
\Delta \varphi=\left(v_{1}-v_{2}\right) t_{\mathrm{d}} \cdot 2 \pi
$$

which was included for $t_{\mathrm{d}}=2 \mu \mathrm{s}$. The deviation $\delta(\Delta \bar{v})$ decreases in magnitude with increasing splitting $\Delta v$. It should be noted, that the splitting $\Delta v$ may appear too large or too small. In Fig. 7 we included results for other intensity ratios $r$, line width $\Delta v_{1 / 2}$ the delay time $t_{\mathrm{d}}$. As could be expected from Fig. 5 the variation of $r$ in the range $0.3 \leq r \leq 1$ is only of slight influence. For 
smaller $\Delta v_{1 / 2}$ the deviation $\delta(\Delta \bar{v})$ damps out more rapidly. For shorter $t_{\mathrm{d}}$ the deviation persists for larger $\Delta v$.

\section{Appearence of Lines}

In Figs. 8 and 9 we reproduce the lineshapes of a doublet and a triplet, respectively. The only differences between a) and b) are different values of $\Delta \varphi$ which may arise from different delay times $t_{\mathrm{d}}$. All other parameters remain unchanged. A large variation of the line pattern can be observed. One component of the triplet may vanish. Further examples are given in [15].

\section{Conclusion}

It was shown that the peak frequencies $\bar{v}_{\mathrm{i}}$ of the power spectra, and also of the amplitude spectra, which are $\sqrt{P_{k}}$, are influenced in several ways. Therefore these spectra must be analyzed with great care, a fact which was overlooked in the early period of MWFT spectroscopy. To overcome the difficulties we have chosen two ways.

[1] J. Ekkers and W. H. Flygare, Rev. Sci. Instrum. 47, 448 (1976).

[2] J. C. McGurk, T. G. Schmalz, and W. H. Flygare, Adv. Chem. Phys. 25.

[3] H. Dreizler, Mol. Phys. 59, 1 (1986).

[4] D. Ziessow, On-line Rechner in der Chemie, de Gruyter, Berlin 1974, p. 110

[5] Discussion with P. Cox, Bristol, Tour 1983.

[6] W. H. Stolze and D. H. Sutter, Z. Naturforsch. 39a, 1092 (1984).

[7] 1. c. [4], Chapter 2.2 .

[8] E. O. Brigham, FFT Schnelle Fourier-Transformation, Oldenbourg, München 1982, Chapter 5, 6.
The first [16] was to simulate a time domain signal by a sum of signals of type (1). The Doppler half-width was introduced additionally. The signal was Fourier transformed and visually compared with the measured spectrum on the computer screen. This method depends on subjective judgement and is time consuming.

This method was replaced by fitting the experimental time domain signal by a synthesized one as described in a following publication [17]. This method needs long calculation time but it works automatically.

Further the edge region of the spectral range should be avoided.

\section{Acknowledgements}

We thank Dr. P. Cox, Bristol, for rising the question and the members of our group for many discussions, especially N. Heineking for calling our attention to the fact that distortions in triplets can be that large that one line may vanish, and Prof. Dr. H. Mäder for critically reading the manuscript. The Deutsche Forschungsgemeinschaft, the Land Schleswig-Holstein and the Fonds der Chemie provided the fund for developing MWFT spectroscopy.

[9] 1.c. [2] and [3].

[10] l.c. [4], p. 52.

[11] l.c. [4], p. 70, 1.c. [8], Chapter 7.

[12] l.c. [4], p. 63.

[13] W. Stahl, G. Bestmann, H. Dreizler, U. Andresen, and R. Schwarz, Rev. Sci. Instrum. 56, 1759 (1985).

[14] 1.c. [4], p. 67.

[15] O. Böttcher and D. H. Sutter, Z. Naturforsch. 43a, 47 (1988).

[16] I. Merke, Diplom thesis, Kiel 1986.

[17] J. Haekel and H. Mäder, Z. Naturforsch. 43a, 203 (1988). 